PROCEEDINGS OF THE

AMERICAN MATHEMATICAL SOCIETY

Volume 132, Number 2, Pages 455-461

S 0002-9939(03)07069-2

Article electronically published on July 31, 2003

\title{
THE APPROXIMATION ORDER OF POLYSPLINES
}

\author{
OGNYAN KOUNCHEV AND HERMANN RENDER
}

(Communicated by David Sharp)

\begin{abstract}
We show that the scaling spaces defined by the polysplines of order $p$ provide approximation order $2 p$. For that purpose we refine the results on one-dimensional approximation order by $L$-splines obtained by de Boor, DeVore, and Ron (1994).
\end{abstract}

\section{INTRODUCTION}

In the last decade the approximation order of shift-invariant subspaces of the space $L^{2}\left(\mathbb{R}^{n}\right)$ of all square-integrable functions on the euclidean space $\mathbb{R}^{n}$ has been investigated extensively, e.g., in the survey paper [10] approximately 100 references are given. The problem can be formulated in a rather general way: suppose that $\left(V_{h}\right)_{h \in I}$ is a family of subspaces of $L^{2}\left(\mathbb{R}^{n}\right)$ (not necessarily shift-invariant) where $I$ is a subset of $(0, \infty)$ having 0 as an accumulation point. One has to estimate the rates of decay of the approximation error

$$
E\left(f, V_{h}\right):=\inf \left\{\|f-s\|_{L^{2}\left(\mathbb{R}^{n}\right)}: s \in V_{h}\right\}
$$

for $h$ tending to 0 . If $W$ is a subspace of $L^{2}\left(\mathbb{R}^{n}\right)$ endowed with a norm $\|\cdot\|_{W}$, we say that $\left(V_{h}\right)_{h \in I}$ provides approximation order $m$ with respect to the norm $\|\cdot\|_{W}$ if there exists a constant $c_{W}$ such that for every $f \in W$ and for every $h \in I$

$$
E\left(f, V_{h}\right) \leq c_{W} \cdot h^{m} \cdot\|f\|_{W} .
$$

Usually $W$ is the potential space $W_{2}^{m}\left(\mathbb{R}^{n}\right)$ for $m \in(0, \infty)$ defined as the subspace of those $f \in L^{2}\left(\mathbb{R}^{n}\right)$ such that

$$
\|f\|_{W_{2}^{m}\left(\mathbb{R}^{n}\right)}:=(2 \pi)^{-\frac{n}{2}}\left\|(1+|\xi|)^{m} \widehat{f}(\xi)\right\|_{L^{2}\left(\mathbb{R}^{n}\right)}<\infty .
$$

In this note we want to prove that cardinal polysplines of order $p$ provide approximation order $2 p$.

The motivation for the present work comes from the fact that polysplines are useful for solving multivariate interpolation problems [4], [5], [6] and they are of importance for the multivariate Wavelet Analysis; cf. the monograph [9. Recall that a function $S: \mathbb{R}^{n} \backslash\{0\} \rightarrow \mathbb{C}$ is called a cardinal polysplind 1 (on annuli) of

Received by the editors April 5, 2002 and, in revised form, October 2, 2002.

2000 Mathematics Subject Classification. Primary 41A15; Secondary 35J40, 31B30.

Key words and phrases. Cardinal splines, cardinal $L$-splines, polysplines, approximation order of splines, polyharmonic functions, cardinal polysplines.

1 The first author introduced polysplines in 1991 in a more general setting with arbitrary interfaces; see [3] and [9].

(C)2003 American Mathematical Society 
order $p$ if $S$ is $(2 p-2)$-times continuously differentiable and the restriction of $S$ to each open annulus $\left\{x \in \mathbb{R}^{n}: e^{l}<|x|<e^{l+1}\right\}$ is a polyharmonic function ${ }^{2}$ of order $p$ for $l \in \mathbb{Z}$. The reason for calling such polysplines "cardinal" is found in Theorem 3 , where it is seen that after expanding $S$ in a Fourier-Laplace series of spherical harmonics, the coefficients $S_{k, l}(\log r)$ are cardinal $L$-splines in the usual sense of the word; cf. Micchelli's paper of 1976 .

Introducing a parameter $h>0$, by $P_{h}$ we denote the set of all functions $S$ : $\mathbb{R}^{n} \backslash\{0\} \rightarrow \mathbb{C}$ which are $(2 p-2)$-times continuously differentiable and whose restriction to each open annulus $A_{h, l}:=\left\{x \in \mathbb{R}^{n}: e^{h l}<|x|<e^{h(l+1)}\right\}$ is a polyharmonic function of order $p$ for $l \in \mathbb{Z}$. Then the scaling spaces of polysplines of order $p$, in short $P V_{h}$, are defined as the $L^{2}$-closure of $P_{h} \cap L^{2}\left(\mathbb{R}^{n}\right), h>0$.

The main result is the following:

Theorem 1. The sequence $\left(P V_{h}\right)_{h>0}$ provides approximation order $2 p$ where $p$ denotes the order of the polysplines. More precisely, there exists a constant $C>0$ such that for all $h$ with $0<h<1$ and $f \in L^{2}\left(\mathbb{R}^{n}\right)$ the following inequality holds:

$$
\inf \left\{\|f-g\|_{L^{2}\left(\mathbb{R}^{n}\right)}: g \in P V_{h}\right\} \leq C \cdot h^{2 p} \cdot\left(\left.\left.\int_{\mathbb{R}^{n}}|| x\right|^{2 p} \cdot \Delta^{p} f(x)\right|^{2} d x\right)^{\frac{1}{2}} .
$$

Note that in place of the norm (3) we have a semi-norm on the right-hand side which is zero on the polyharmonic functions of order $p$.

The paper is organized as follows: in Section 2 we discuss the approximation order of cardinal $L$-splines by using important results from 2. In Section 3 the main result will be proven.

\section{Approximation ORDER of CARDinal $L$-SPLines}

Let us recall Theorem 4.3 in the fundamental paper 2]: Suppose that for every $h>0$, the space $S_{h}$ is the $L^{2}\left(\mathbb{R}^{n}\right)$-closure of the linear space generated by the shifts $\varphi_{h}(\cdot-m), m \in \mathbb{Z}^{n}$ of the function $\varphi_{h} \in L^{2}\left(\mathbb{R}^{n}\right)$ (so $S_{h}$ is the shift-invariant space generated by $\left.\varphi_{h}\right)$ and that $V_{h}=\left\{s(\dot{\bar{h}}): s \in S_{h}\right\}$. Then the family $\left(V_{h}\right)_{h \in I}$ provides approximation order $m$ with respect to the norm $\|\cdot\|_{W_{2}^{m}\left(\mathbb{R}^{n}\right)}$ defined in (3) if and only if there exists $D>0$ such that for all $h \in I$ and for almost all $x \in C:=[-\pi, \pi]^{n}$

$$
\left|\Lambda_{\varphi_{h}}(x)\right| \leq D \cdot\left(h+|x|^{m}\right),
$$

where

$$
\left(\Lambda_{\varphi_{h}}(\xi)\right)^{2}:=\frac{\sum_{\alpha \in \mathbb{Z}^{n}, \alpha \neq 0}\left|\widehat{\varphi_{h}}(\xi+2 \pi \alpha)\right|^{2}}{\sum_{\beta \in \mathbb{Z}^{n}}\left|\widehat{\varphi_{h}}(\xi+2 \pi \beta)\right|^{2}} \leq 1 .
$$

We will need a refinement of that result. For our purposes it will be useful to consider, instead of (3), different norms. In the following we replace the function $(1+|x|)^{m}$ by a measurable function $Q(x)$ with the following properties: (i) the zero set $Q^{-1}(0)$ of $Q$ is a set of Lebesgue measure zero and (ii) there exists a constant $D_{1}>0$ such that

$$
\left|Q\left(\frac{x}{h}\right)\right| \geq D_{1} \frac{1}{h^{m}} \quad \text { for all } x \notin C:=[-\pi, \pi]^{n} .
$$

\footnotetext{
${ }^{2}$ Recall that a function $f$ defined on an open set $U$ in the euclidean space $\mathbb{R}^{n}$ is polyharmonic of order $p$ if $f$ is $2 p$-times continuously differentiable and $\Delta^{p} f(x)=0$ for all $x \in U$ where $\Delta$ is the Laplace operator and $\Delta^{p}$ its $p$-th iterate.
} 
Suppose further that there exists a constant $D_{2}>0$ such that for all $x \in C$ and for all $0<h<1$

$$
\left|\Lambda_{\varphi_{h}}(h x)\right| \leq h^{m} D_{2}|Q(x)| .
$$

An analysis of the proof in [2] shows that then the following inequality holds (for us the constants $D_{1}$ and $D_{2}$ defined in the formula will be very important!)

$$
E\left(f, V_{h}\right) \leq\left(D_{2}(2 \pi)^{\frac{n}{2}}+\frac{1}{D_{1}(2 \pi)^{\frac{n}{2}}}\right) \cdot h^{m} \cdot\|Q(\xi) \widehat{f}(\xi)\|_{L_{2}\left(\mathbb{R}^{n}\right)} .
$$

We recall some facts about $L$-splines: Let $L$ be a linear differential operator with constant coefficients of order $N+1$, say

$$
L=M_{\Lambda}:=\prod_{j=1}^{N+1}\left(\frac{d}{d v}-\lambda_{j}\right) \text { where } \Lambda:=\left(\lambda_{1}, \ldots, \lambda_{N+1}\right) .
$$

Then a function $u: \mathbb{R} \rightarrow \mathbb{R}$ is called a cardinal L-spline on the mesh $h \mathbb{Z}(h>0)$ if $u$ is $(N-1)$-times continuously differentiable and if for every $l \in \mathbb{Z}$ there exists $f_{l} \in U_{L}:=\left\{f \in C^{\infty}(\mathbb{R}): L f=0\right\}$ such that $u(t)=f_{l}(t)$ for all $t \in(l h,(l+1) h)$. The set of all cardinal $L$-splines for the operator $L=M_{\Lambda}$ on $h \mathbb{Z}$ will be denoted by $\mathcal{S}_{h \mathbb{Z}}(\Lambda)$. The scaling spaces $V_{h}(\Lambda)$ are defined by

$$
V_{h}(\Lambda)=L^{2}(\mathbb{R}) \text {-closure of } \mathcal{S}_{h \mathbb{Z}}(\Lambda) \cap L^{2}(\mathbb{R}) .
$$

Let $Q_{\Lambda}$ be the basic spline which can be defined by its Fourier transform by

$$
\widehat{Q_{\Lambda}}(\xi)=\frac{\prod_{j=1}^{N+1}\left(e^{-\lambda_{j}}-e^{-i \xi}\right)}{\prod_{j=1}^{N+1}\left(i \xi-\lambda_{j}\right)} .
$$

Theorem 2. Let $N \in \mathbb{N}$ be fixed. Then there exists a constant $D>0$ such that for all $\Lambda=\left(\lambda_{1}, \ldots, \lambda_{N+1}\right) \in \mathbb{R}^{N+1}$ and for all $f \in L_{2}(\mathbb{R})$ the following inequality holds:

$$
E\left(f, V_{h}(\Lambda)\right) \leq h^{N+1} \cdot D\left\|P_{\Lambda}(\xi) \widehat{f}(\xi)\right\|_{L_{2}(\mathbb{R})},
$$

where the polynomial $P_{\Lambda}(x)=\prod_{j=1}^{N+1}\left(i x-\lambda_{j}\right)$.

Remark 3. Note that if we used the usual Sobolev norm (3), then we would not be able to obtain the sharp constant $D$ of inequality (11); the last is the main virtue of Theorem 2 ,

Proof. By the above we have to check (5) and (6). Note that for $Q:=P_{\Lambda}$ we have the estimate

$$
\left|P_{\Lambda}\left(\frac{x}{h}\right)\right|^{2}=\prod_{j=1}^{N+1}\left(\left(\frac{x}{h}\right)^{2}+\lambda_{j}^{2}\right) \geq \pi^{2(N+1)} \frac{1}{h^{2(N+1)}}
$$

for all $|x| \geq \pi$ and for all $h>0$. Hence it suffices to show that

$$
\left|\Lambda_{\varphi_{h}}(h \xi)\right|^{2} \leq h^{2(N+1)}\left|P_{\Lambda}(\xi)\right|^{2} \sum_{\alpha \in \mathbb{Z}, \alpha \neq 0} \frac{1}{(\pi|\alpha|)^{2(N+1)}} .
$$


The trivial inequality $\left(\Lambda_{\varphi_{h}}(\xi)\right)^{2} \leq \frac{\sum_{\alpha \in \mathbb{Z}, \alpha \neq 0}\left|\widehat{\varphi_{h}}(\xi+2 \pi \alpha)\right|^{2}}{\left|\widehat{\varphi_{h}}(\xi)\right|^{2}}$ and the estimate

$$
\frac{\left|\widehat{\varphi_{h}}(\xi+2 \pi \alpha)\right|^{2}}{\left|\widehat{\varphi_{h}}(\xi)\right|^{2}}=\frac{\left|\widehat{Q_{h \Lambda}}(\xi+2 \pi \alpha)\right|^{2}}{\left|\widehat{Q_{h \Lambda}}(\xi)\right|^{2}}=\prod_{j=1}^{N+1}\left|\frac{i \xi-h \lambda_{j}}{i(\xi+2 \pi \alpha)-h \lambda_{j}}\right|^{2}
$$

yield

$$
\left|\Lambda_{\varphi_{h}}(h \xi)\right|^{2} \leq h^{2(N+1)} \prod_{j=1}^{N+1}\left(\xi^{2}+\lambda_{j}^{2}\right) \sum_{\alpha \in \mathbb{Z}, \alpha \neq 0} \prod_{j=1}^{N+1} \frac{1}{(h \xi+2 \pi \alpha)^{2}+h^{2} \lambda_{j}^{2}} .
$$

Since $(h \xi+2 \pi \alpha)^{2}+h^{2} \lambda_{j}^{2} \geq(h \xi+2 \pi \alpha)^{2} \geq(2 \pi|\alpha|-|h \xi|)^{2}$ we obtain for $0<h<1$ and $|\xi| \leq \pi$ the estimate $2 \pi|\alpha|-|h \xi| \geq \pi|\alpha|$ (since $\alpha \neq 0$ ) arriving at (13)).

\section{ThE APPROXIMATION ORDER OF POLYSPLINES}

Let $\mathbb{S}^{n-1}=\left\{x \in \mathbb{R}^{n} ;|x|=1\right\}$ be the unit sphere. Each $x \in \mathbb{R}^{n}$ will be written in spherical coordinates $x=r \theta$ with $r \geq 0$ and $\theta \in \mathbb{S}^{n-1}$. Recall that a function $Y: \mathbb{S}^{n-1} \rightarrow \mathbb{C}$ is a spherical harmonic of degree $k \in \mathbb{N}_{0}$ if there exists a homogeneous harmonic polynomial $P(x)$ of degree $k$ such that $P(\theta)=Y(\theta)$ for all $\theta \in \mathbb{S}^{n-1}$. The set $\mathfrak{H}_{k}$ of all spherical harmonics of degree exactly $k$ is a linear space of dimension $a_{k}:=\operatorname{dim} \mathfrak{H}_{k}=\left(\begin{array}{c}n+k-1 \\ k\end{array}\right)-\left(\begin{array}{c}n+k-3 \\ k-2\end{array}\right)$. We denote by $Y_{k, l}$ with $l=1,2, \ldots, a_{k}$ a basis for $\mathfrak{H}_{k}$. For a detailed account we refer to Stein and Weiss [12].

Let $u:\left(R_{1}, R_{2}\right) \rightarrow \mathbb{C}$ be infinitely differentiable and $Y_{k} \in \mathfrak{H}_{k}$. Then it is well known that $\Delta\left(u(r) Y_{k}(\theta)\right)=Y_{k}(\theta) L_{(k)} u(r)$ where we have put

$$
L_{(k)}=\frac{d^{2}}{d r^{2}}+\frac{n-1}{r} \frac{d}{d r}-\frac{k(k+n-2)}{r^{2}} .
$$

By iteration we have $\Delta^{p} u=Y_{k}(\theta) \cdot\left[L_{(k)}\right]^{p} u(r)$. For convenience, we write

$$
\begin{aligned}
& \Lambda_{+}(k, p):=\{k, k+2, \ldots, k+2 p-2\}, \\
& \Lambda_{-}(k, p):=\{-k-n+2,-k-n+4, \ldots,-k-n+2 p\} .
\end{aligned}
$$

The space of solutions of the equation $L_{(k)}^{p} f(r)=0$ which are $C^{\infty}$ for $r>0$ is generated by a simple basis: for $j \in \Lambda_{+}(k, p) \cup \Lambda_{-}(k, p)$ the function $r^{j}$ is clearly a solution, while for $j \in \Lambda_{+}(k, p) \cap \Lambda_{-}(k, p)$ we obtain a second solution $r^{j} \log r$. It will be convenient to make a transform of the variable $r$ to $v=\log r$. Then a solution of the form $r^{j}$ will be transformed to $e^{j v}$ and a solution of the form $r^{j} \log r$ is transformed to $v e^{j v}$. We see immediately that all solutions to the equation $L_{(k)}^{p} f(r)=0$ are transformed to solutions of the equation $M_{\Lambda(k)} g(v)=0$ where $M_{\Lambda(k)}$ is defined by (8) with respect to the vector

$$
\Lambda_{k}:=(k, k+2, \ldots, k+2(p-1),-(k+n)+2, \ldots,-(k+n)+2 p) .
$$

The dependence on the parameter $p$ and $n$ will be suppressed throughout the paper.

A proof of the following can be found in [6] and [9, Theorem 9.7].

Theorem 4. Let $S: \mathbb{R}^{n} \backslash\{0\} \rightarrow \mathbb{R}$ be a polyspline of order $p$. Then the LaplaceFourier coefficient $S_{k, l}: \mathbb{R} \rightarrow \mathbb{R}$ defined by

$$
S_{k, l}(v):=\int_{\mathbb{S}^{n-1}} S\left(e^{v} \theta\right) Y_{k, l}(\theta) d \theta
$$

is a cardinal L-spline with respect to the linear differential operator $M_{\Lambda(k)}$. 
We want to characterize the $L^{2}\left(\mathbb{R}^{n}\right)$-closure $P V_{h}$. It is a temptation to assume that for $S \in P V_{h}$ the Fourier-Laplace coefficient defined through formula (16) will be in $V_{h}\left(\Lambda_{k}\right)$, i.e., in the closure of $\mathcal{S}_{h \mathbb{Z}}\left(\Lambda_{k}\right) \cap L_{2}(\mathbb{R})$. This is not true since the transformation rule will give us an additional weight for $f \in L_{2}\left(\mathbb{R}^{n}\right)$ :

$$
\int_{\mathbb{R}^{n}}|f(x)|^{2} d x=\int_{0}^{\infty} \int_{\mathbb{S}^{n-1}}|f(r \theta)|^{2} r^{n-1} d \theta d r .
$$

Fortunately, this problem can be easily solved; see e.g. 7].

Theorem 5. Define $\overline{\Lambda_{k}}=\left(\frac{n}{2}, \ldots, \frac{n}{2}\right)+\Lambda_{k}$. Then for each $k \in \mathbb{N}_{0}, l=1, \ldots, a_{k}$, the following map, defined on $P_{h} \cap L^{2}\left(\mathbb{R}^{n}\right)$ by

$$
S \longmapsto \bar{S}_{k, l}(v):=e^{\frac{n}{2} v} \int_{\mathbb{S}^{n-1}} S\left(e^{v} \theta\right) Y_{k, l}(\theta) d \theta,
$$

maps onto $\mathcal{S}_{h \mathbb{Z}}\left(\overline{\Lambda_{k}}\right) \cap L^{2}(\mathbb{R}, d v)$, and, by continuity, it can be extended to a map from $P V_{h}$ onto $V_{h}\left(\overline{\Lambda_{k}}\right)$. Furthermore, $P V_{h}$ is isomorphic to

$$
V_{h}:=\bigoplus_{k \in \mathbb{N}_{0}, l=1, \ldots, a_{k}} V_{h}\left(\overline{\Lambda_{k}}\right) .
$$

Proof of Theorem 1. Let $f \in L^{2}\left(\mathbb{R}^{n}\right)$ and $g \in P V_{h}$. Then by the transformation rule (17),

$$
\|f-g\|_{L^{2}\left(\mathbb{R}^{n}\right)}^{2}=\int_{0}^{\infty} \int_{\mathbb{S}^{n-1}}|f(r \theta)-g(r \theta)|^{2} r^{n-1} d \theta d r .
$$

Let $f_{k, l}$ and $g_{k, l}$ be the Laplace-Fourier coefficients of $f$ and $g$ respectively as defined in (16). Note that $v \longmapsto \overline{g_{k, l}}\left(e^{v}\right):=e^{\frac{n}{2} v} g_{k, l}\left(e^{v}\right)$ is in $V_{h}\left(\overline{\Lambda_{k}}\right)$. Since $Y_{k, l}(\theta)$ constitutes an orthonormal basis, we obtain

$$
\|f-g\|_{L^{2}\left(\mathbb{R}^{n}\right)}^{2}=\sum_{k=0}^{\infty} \sum_{l=1}^{a_{k}} \int_{-\infty}^{\infty}\left|f_{k, l}\left(e^{v}\right)-g_{k, l}\left(e^{v}\right)\right|^{2} e^{n v} d v
$$

Minimizing the expression $g \longmapsto\|f-g\|_{L^{2}\left(\mathbb{R}^{n}\right)}^{2}$ for $g \in P V_{h}$ is equivalent to minimizing the expression

$$
\int_{-\infty}^{\infty}\left|e^{\frac{n}{2} v} f_{k, l}\left(e^{v}\right)-\overline{g_{k, l}}\left(e^{v}\right)\right|^{2} d v
$$

for each $k \in \mathbb{N}, l=1, \ldots, a_{k}$, where $\overline{g_{k, l}} \in V_{h}\left(\overline{\Lambda_{k}}\right)$. Theorem 2 applied to $\Lambda=\overline{\Lambda_{k}}$ (hence $N+1=2 p$ ) shows that there exists a constant $C_{p}>0$ which only depends on $p$ (and not on the values $\lambda_{j}$ in $\overline{\Lambda_{k}}$ ) such that

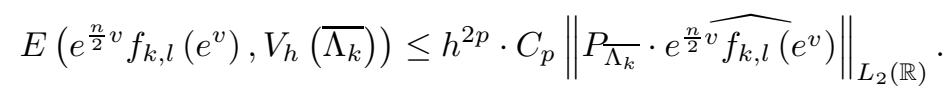

Write $G_{k, l}(v):=e^{\frac{n}{2} v} f_{k, l}\left(e^{v}\right)$. A simple computation (using Parseval's identity and the fact that differentiation becomes multiplication via Fourier transform) shows that

$$
\frac{1}{2 \pi}\left\|P_{\overline{\Lambda_{k}}} \cdot \widehat{G_{k, l}}\right\|_{L_{2}(\mathbb{R})}^{2}=\int_{-\infty}^{\infty}\left|M_{\overline{\Lambda_{k}}} G_{k, l}(v)\right|^{2} d v
$$


A calculation shows that $M_{\overline{\Lambda_{k}}}\left(e^{\frac{n}{2} v} f_{k, l}\left(e^{v}\right)\right)=e^{\frac{n}{2} v} M_{\Lambda_{k}}\left(f\left(e^{v}\right)\right)$. Then (20) and (21) yield

$$
E\left(f, P V_{h}\right)^{2} \leq h^{4 p} \cdot 2 \pi C_{p}^{2} \sum_{k=0}^{\infty} \sum_{l=1}^{a_{k}}\left\|e^{\frac{n}{2} v} M_{\Lambda_{k}}\left(f\left(e^{v}\right)\right)\right\|_{L_{2}\left(\mathbb{R}^{n}\right)}^{2} .
$$

The next theorem applied to the case $p=q$ finishes the proof.

Theorem 6. Let $p, q \in \mathbb{N}_{0}$ and define $\|f(x)\|_{q, p}^{2}:=\left.\left.\int|| x\right|^{2 q} \cdot \Delta^{p} f(x)\right|^{2} d x$ for $f \in L_{2}\left(\mathbb{R}^{n}\right)$. Then

$$
\|f(x)\|_{q, p}^{2}=\sum_{k=0}^{\infty} \sum_{l=1}^{a_{k}} \int\left|e^{v\left(2 q-2 p+\frac{n}{2}\right)} M_{\Lambda_{k}}\left(f_{k, l}\left(e^{v}\right)\right)\right|^{2} d v
$$

where $f_{k, l}(r)$ are the Laplace-Fourier coefficients of $f$ defined as in equality (16).

Proof. Assume that $f(r \theta)=f_{k, \ell}(r) Y_{k, \ell}(\theta)$. Since $\Delta^{p} f(x)=L_{k}^{p} f_{k, \ell}(r) Y_{k, \ell}(\theta)$, we obtain

$$
\|f(x)\|_{q, p}^{2}=\int_{0}^{\infty} \int_{\mathbb{S}^{n-1}}\left|r^{2 q} L_{(k)}^{p} f_{k, \ell}(r) Y_{k, \ell}(\theta)\right|^{2} r^{n-1} d r d \theta .
$$

The integration over $\theta$ only gives a factor 1 . Now we change the variable $r=e^{v}$ and apply the identity $\left(L_{k}^{p} f_{k, l}\right)\left(e^{v}\right)=e^{-2 v p} M_{\Lambda_{k}}\left(f_{k, l}\left(e^{v}\right)\right)$; see e.g. Theorem 10.34 in 9]. Then

$$
\|f(x)\|_{q, p}^{2}=\int\left|e^{2 v q} e^{-2 v p} M_{\Lambda_{k}}\left(f_{k, l}\left(e^{v}\right)\right)\right|^{2} e^{n v} d v .
$$

Finally, we see that for arbitrary $f \in L^{2}\left(\mathbb{R}^{n}\right)$ the result follows via the orthogonal decomposition of $f$ in spherical harmonics.

\section{ACKNOWLEDGEMENT}

The first author was partially sponsored by the Fulbright Program during his stay at the University of Wisconsin-Madison. The second author thanks the Alexander von Humboldt Foundation for supporting him in the framework of the FeodorLynen program.

\section{REFERENCES}

[1] Adams, R., Sobolev Spaces, Academic Press, New York-San Francisco-London, 1975. MR 56:9247

[2] de Boor, C., DeVore, R. A., Ron, A., Approximation from shift-invariant subspaces of $L_{2}\left(\mathbb{R}^{d}\right)$, Trans. Amer. Math. Soc. 341 (1994), pp. 787-806. MR 94d:41028

[3] Kounchev, O. I., Definition and basic properties of polysplines, I and II, C. R. Acad. Bulg. Sci., 44 (1991), nos. 7 and 8, pp. 9-11, pp.13-16. MR 93a:41016 MR 92m:41031

[4] Kounchev, O. I., Render, H., Multivariate cardinal splines via spherical harmonics. Submitted.

[5] Kounchev, O. I., Render, H., The interpolation problem for cardinal splines. Submitted.

[6] Kounchev, O. I., Render, H., Symmetry of interpolation polysplines and L-splines, Trends in Approximation Theory, K. Kopotun, T. Lyche, and M. Neamtu (eds.), Vanderbilt University Press, Nashville, TN, 2001.

[7] Kounchev, O. I., Render, H., Wavelet Analysis of cardinal L-splines and construction of multivariate prewavelets, Proceedings of Tenth Approximation Theory Conference (St. Louis, 2001), Innov. Appl. Math. Vanderbilt Univ. Press, Nashville, TN, 2002, pp. 333-353.

[8] Micchelli, Ch., Cardinal L-splines, In: Studies in Spline Functions and Approximation Theory, Eds. S. Karlin et al., Academic Press, NY, 1976, pp. 203-250. MR 58:1866 
[9] Kounchev, O. I., Multivariate Polysplines. Applications to Numerical and Wavelet Analysis, Academic Press, Boston, 2001. MR 2002h:41001

[10] Jetter, K., Plonka G., A survey on $L_{2}$-approximation order from shift-invariant spaces, In: Multivariate Approximation and Applications (N. Dyn, D. Leviatan, D. Levin, and A. Pinkus, eds.), pp. 73-111. Cambridge University Press, 2001. MR 2001m:65005

[11] Meyer, Y. Wavelets and Operators, Cambridge University Press, 1992. MR 94f:42001

[12] Stein, E. M., Weiss, G., Introduction to Fourier Analysis on Euclidean Spaces, Princeton University Press, Princeton, NJ, 1971. MR 46:4102

Institute of Mathematics, Bulgarian Academy of Sciences, Acad. G. Bonchev St. 8, 1113 Sofia, Bulgaria

E-mail address: kounchev@cblink.net

E-mail address: kounchev@math.uni-duisburg.de

E-mail address: kounchev@math.bas.bg

Institute of Mathematics, University of Duisburg-Essen, Lotharstr. 65, 47048 DuisBURG, Germany

E-mail address: render@math.uni-duisburg.de 